\title{
Downregulation of FoxM1 inhibits proliferation, invasion and angiogenesis of HeLa cells in vitro and in vivo
}

\author{
HONG CHEN, YANG ZOU, HONG YANG, JINGJING WANG and HONG PAN \\ Department of Gynecology and Obstetrics, Zhongnan Hospital of Wuhan University, Wuhan, Hubei 430071, P.R. China
}

Received June 30, 2014; Accepted August 14, 2014

DOI: $10.3892 / \mathrm{ijo} .2014 .2645$

\begin{abstract}
FoxM1 is a specific transcription factor that has an important function in aggressive human carcinomas, including cervical cancer. However, the specific function and internal molecular mechanism in cervical cancer remain unclear. In this study, RNAi-mediated FoxM1 knockdown inhibited cell growth. This process also decreased the migration and invasion activities of HeLa cells in vitro. Downregulation of FoxM1 inhibited tumor growth and angiogenesis in vivo. In addition, the expressions of uPA, matrix metalloproteinase (MMP)-2, MMP-9 and VEGF were significantly decreased in vitro and in vivo. These results suggested that the inactivation of FoxM1 could be a novel therapeutic target for cervical cancer treatment.
\end{abstract}

\section{Introduction}

Cervical cancer, a potentially preventable disease with a high incidence, remains the second most common malignancy in women worldwide (1). Approximately one-third of patients who manifest invasive cervical cancer die because of this disease (2). The onset of this disease occurs in young individuals to a high extent (3). Although advanced surgical techniques and chemoradiotherapy can improve the treatment rate of cervical cancer, mortality rate remains high because of tumor recurrence and drug resistance in chemoradiotherapy (4). As such, novel targets required for cervical cancer treatment should be developed.

Forkhead box protein M1 (FoxM1) is a specific transcription factor that belongs to a family of evolutionarily conserved proteins characterized by the presence of a DNA-binding domain called the forkhead box (5). The aberrant expression and function of FoxM1 have been verified in carcinoma progression and malignant carcinomas, such as lung cancer (6), breast cancer (5), glioblastoma (7), pancreatic cancer (8),

Correspondence to: Dr Hong Chen, Department of Gynecology and Obstetrics, Zhongnan Hospital of Wuhan University, Wuhan, Hubei 430071, P.R. China

E-mail: znchgg@163.com

Key words: FoxM1, migration, invasion, tumorigenesis, angiogenesis gastric cancer (9), hepatocellular carcinoma (10) and cervical cancer $(11,12)$. FoxM1 is also known as a dynamic cancerassociated biomarker involved in cell cycle progression, differentiation, DNA damage repair, angiogenesis and other biological processes (13-20).

In our previous study, the total expression of FoxM1 in cervical cancer tissues is higher than that in normal cervical tissues, and the nuclear expression of FoxM1 is evidently correlated with pathological stages (12). These results demonstrate that FoxM1 has been linked to tumorigenesis and progression. FoxM1 also participates in or stimulates other biological behaviors of tumors, such as angiogenesis, invasion and metastasis. Studies have simultaneously demonstrated that the expression of FoxM1 is positively correlated with urokinase-type PA (uPA), matrix metalloproteinase (MMP)-2 and MMP-9 expressions, resulting in the degradation of the extracellular matrix, migration and invasion of tumor cells $(5,8)$. Another biomarker, VEGF is involved in angiogenesis and tumor growth; this biomarker has also been implicated in tumor progression (17,21). Hence, FoxM1 signalling possibly regulates tumor progression with these essential factors in cervical cancer.

To elucidate this information, we applied RNAi technique and evaluated the function of FoxM1 on the proliferation, apoptosis, migration and invasion of HeLa cells as well as tumorigenesis and angiogenesis in nude mice. The results may provide evidence for the molecular-targeted therapy of cervical cancer.

\section{Materials and methods}

Cell culture. Human cervical cancer cell lines, including HeLa, SiHa and C33A, used in the present study were generously provided by the Scientific Research Center in Zhongnan Hospital of Wuhan University. The cells were maintained in DMEM (Hyclone, China) containing $10 \%$ fetal bovine serum, $1 \%$ penicillin and streptomycin in a humid $5 \% \mathrm{CO}_{2}$ atmosphere at $37^{\circ} \mathrm{C}$.

Plasmid and stable transfection. Human FoxM1-specific RNAi plasmid vectors that express shRNAs or empty vectors were purchased from Shanghai Genechem Co. Ltd. (Shanghai, China). All of the vectors were expressed under the control of a CMV promoter. In brief, $2.0 \times 10^{5} \mathrm{HeLa}$ cells/lane were seeded in a 6-well culture plate and transfected with the appro- 
priate plasmids by using Lipofectamine 2000 (Invitrogen, USA) according to the manufacturer's instructions. The cells were incubated at $37^{\circ} \mathrm{C}$ for $6 \mathrm{~h}$. Afterwards, lipid and plasmid complexes were removed and a fresh medium was added. At $48 \mathrm{~h}$ after transfection, stable transfectants were selected from $700 \mu \mathrm{g} / \mathrm{ml} \mathrm{G} 418$ for four weeks. Individual clones were isolated using pipette tips and maintained in G418 (350 $\mu \mathrm{g} / \mathrm{ml})$.

Cell proliferation by MTT assay and cell apoptosis by TUNEL assay. We applied MTT assay to determine cell viability. The number of cells was counted every $24 \mathrm{~h}$ for 7 days. A TUNEL apoptosis detection kit (Promega) was used for DNA fragmentation fluorescence staining according to the manufacturer's protocol. Positively stained, fluorescein-labelled cells were visualised and counted using a fluorescence microscope (Nikon Eclipse 80i, China).

Immunofluorescence analysis. In immunofluorescent staining, the cells were fixed with $4 \%$ formaldehyde, blocked for $30 \mathrm{~min}$ in $1 \%$ BSA prepared in PBS and incubated overnight in primary antibody at a concentration of $1: 100$ at $4^{\circ} \mathrm{C}$. The cells were subsequently incubated in appropriate fluorescencelabelled secondary antibody for $1 \mathrm{~h}$ at room temperature. The slides were then mounted with DAPI (Beyotime, China) to visualize the nucleus. Fluorescent photomicrographs were obtained using a fluorescence microscope.

ELISA for $u P A, M M P-2, M M P-9$ and VEGF. The assays were assessed using uPA, MMP-2, MMP-9 and VEGF ELISA kits according to the manufacturer's protocol. The cultivated cells were incubated in 6-well plates for $24 \mathrm{~h}$. UPA, MMP-2, MMP-9 and VEGF concentrations were measured using the corresponding ELISA kits (Elabscience, China), afterwards, the culture medium was collected and centrifuged to remove cell debris.

Cell migration and invasion assays. Cell migration and invasion assays were obtained using 24 -well chambers $(8-\mu \mathrm{m}$ pore size) with or without Matrigel according to the manufacturer's protocol. The cells that penetrated the membrane were determined by counting the mean cell number of five x20 magnification fields randomly and photographed under an inverted phase-contrast microscope (Nikon Eclipse 80i). These experiments were repeated in triplicate.

Xenograft experiments. Nude mice (BALB/c nu/nu, females; 4-5-week-old) were purchased from the Laboratory Animal Center of Wuhan University and housed under SPF conditions. The experimental protocols were approved by the Animal Research Committee of Zhongnan Hospital of Wuhan University. The nude mice were randomly assigned to three groups. FoxM1-shRNA, empty vector and parental HeLa cells suspended in PBS were inoculated subcutaneously on the right oxter with $1 \times 10^{7}$ cells. Tumor growth was measured at an interval of 6 days after injection by using a calliper, and tumor volume was calculated according to the following formula: length $\mathrm{x}$ width ${ }^{2} \mathrm{x} 0.5$ (22). All the mice were euthanized at day 35 post-inoculation. Harvested tumor tissues were removed from each mouse, weighed and cut into two parts. One part was placed in liquid nitrogen and then frozen at $-80^{\circ} \mathrm{C}$; the remaining part was fixed in $10 \%$ buffered formalin, embedded in paraffin, sectioned and stained.

Real-time quantitative PCR ( $q P C R$ ) assay. Total RNA was extracted from the cultured cells or tumor xenografts by using TRIzol. The purity of the extracted RNA was then determined by spectrophotometry. The designed premier sequences of uPA, MMP-2, MMP-9, VEGF and $\beta$-actin genes are shown in Table I. The first-strand cDNA was synthesized from $4.823 \mu \mathrm{g}$ of total purified mRNA in 96-well plates with these primers in a total volume of $20 \mu \mathrm{l}$. The targeted cDNAs were amplified using SYBR Green Master Mix. The PCR conditions of the genes included the following: one cycle of $50^{\circ} \mathrm{C}$ for $2 \mathrm{~min}$ and $95^{\circ} \mathrm{C}$ for $10 \mathrm{~min} ; 40$ cycles of $95^{\circ} \mathrm{C}$ for $30 \mathrm{sec}$ and $60^{\circ} \mathrm{C}$ for $30 \mathrm{sec}$.

Western blot assay. Tumor protein was extracted from cells and tumor xenografts by using RIPA buffer. Equal amounts of protein $(50 \mu \mathrm{g} / \mathrm{lane})$ were electrophoresed on SDS-PAGE gels and blotted on a PVDF membrane (Millipore). Rabbit antiFoxM1 (1:500, Santa, China), anti-uPA (1:500, Santa, China), anti-MMP2 (1:600, Bioworld, China), anti-MMP9 (1:600, Bioworld), anti-VEGF (1:500, Abcam, China) and $\beta$-actin (1:1,000, Boster, China) were used as primary antibodies. HRP-conjugated goat anti-rabbit (1:50,000, Boster) was used as a secondary antibody. The protein bands were detected on $\mathrm{X}$-ray film by using an enhanced chemiluminescence detection system.

Microvessel density test. To compare the number of capillaries that formed, we immunohistochemically analyzed the serial sections from xenograft tumors of each group. The sections were blocked with $3 \% \mathrm{H}_{2} \mathrm{O}_{2}$ at room temperature for $15 \mathrm{~min}$ and the slides were incubated with primary antibody CD31, which is considered as an endothelial cell-specific marker (1:200, Bioworld), overnight at $4^{\circ} \mathrm{C}$. Secondary antibodies biotin-labelled anti-rabbit IgG (Bioworld) were used to visualize the specific markers by avidin-HRP/DAB reaction. Negative controls were obtained by replacing the primary antibody with PBS. Microvessel density (MVD) was quantified by observing the number of vessels and immunoreactive endometrial cells per field at x100 high-power magnification in four vascular 'hot spots', and other details were conducted as described previously (23).

Statistical analysis. Data were expressed as mean \pm SD from at least three separate experiments. Statistical analysis was performed using GraphPad Prism 5 software and SPSS 13.0 software. The statistical significance of differences was determined by Student's two-tailed t-test in two groups and one-way ANOVA in multiple groups. $\mathrm{P}<0.05$ was considered statistically significant.

\section{Results}

Plasmid vector stably expressing FoxM1 shRNA effectively suppresses FoxM1 expression. In advance, the baseline expression of FoxM1 in a panel of cervical cells in our laboratory was determined by western blot analysis. The highest expression of FoxM1 was observed in HeLa cells, which were then 
Table I. The designed premier sequences in qPCR.

\begin{tabular}{lll}
\hline Abbreviations & \multicolumn{1}{c}{ Forward } & Reverse \\
\hline FoxM1 & 5'-CAACTCAGCCTCCAGGACTC-3' & 5'-CTGCCTCACCATCACAGGTC-3' \\
uPA & 5'-CAGGCGTCTACACGAGAGTC-3' & 5'-TGGCACAGGCAAATCCATCT-3' \\
MMP-2 & 5'-GATAACCTGGATGCCGTCGT-3' & 5'-CGAAGGCAGTGGAGAGGAAG-3' \\
MMP-9 & 5'-CGACGTCTTCCAGTACCGAG-3' & 5'-TTGTATCCGGCAAACTGGCT-3' \\
VEGF & 5'-GGTGCCCGCTGCTGTCTAAT-3' & 5'-GAGATCTGGTTCCCGAAACCC-3' \\
$\beta$-actin & 5'-CACGATGGAGGGGCCGGACTCATC-3' & 5'-TAAAGACCTCTATGCCAACACAGT-3'
\end{tabular}

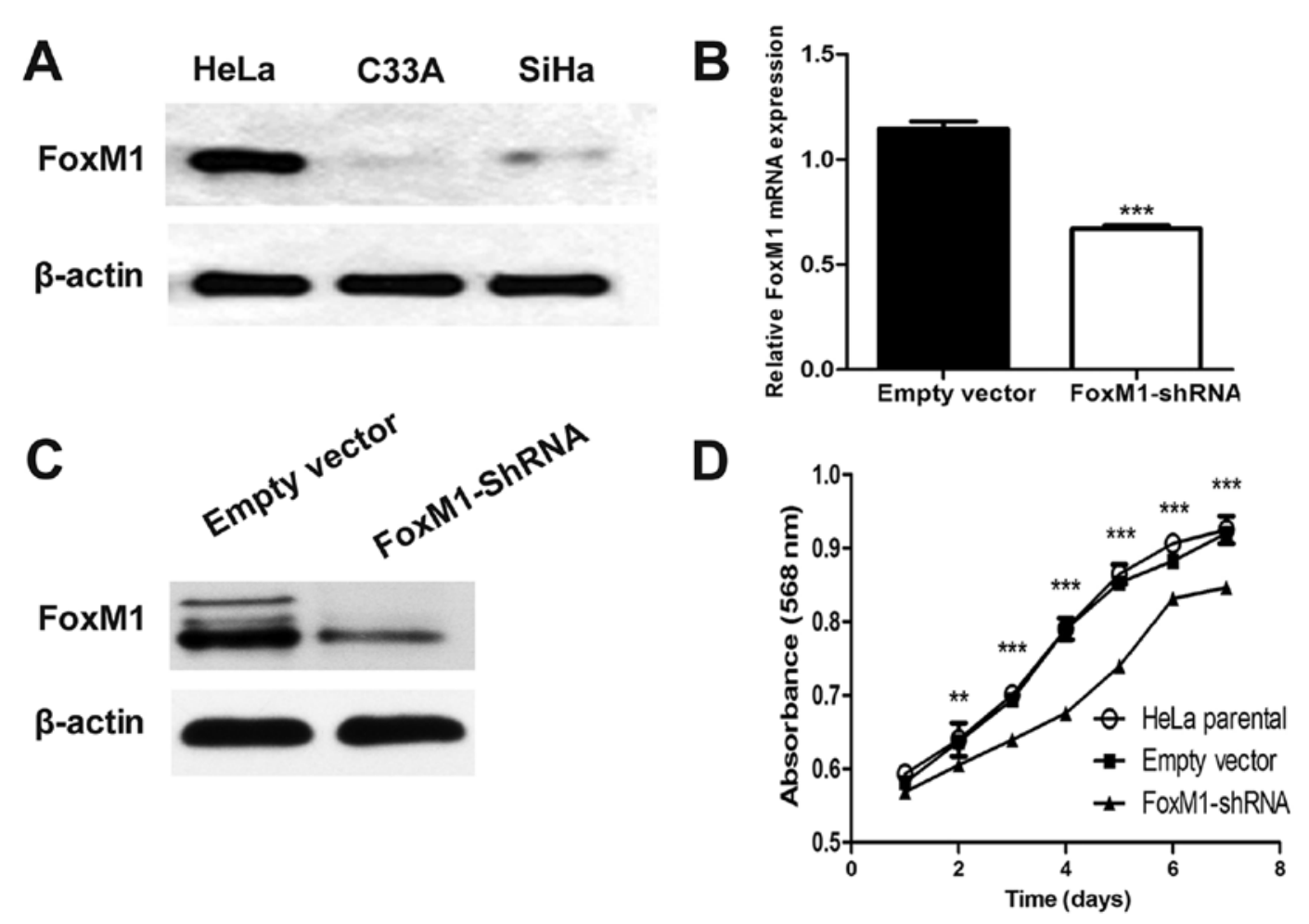

Figure 1. Expression level of FoxM1 protein in different cervical cancer cell lines and the corresponding effect on cell viability by FoxM1-shRNA. (A) FoxM1 protein levels in HeLa, SiHa and C33A cell lines were examined by western blot analysis. (B and C) Interfering effects of shFoxM1 in HeLa were detected using qPCR and western blot analysis. (D) Downregulation of FoxM1 attenuated the proliferation of HeLa cells compared with the empty vector-transfected cells and HeLa parental cells determined by MTT assay. Data are shown as mean \pm SEM. Experiments were repeated thrice.

used in our study (Fig. 1A). After FoxM1-shRNA transfection was performed, FoxM1 expression was remarkably decreased as revealed by qPCR and western blot analysis compared with empty vector-transfected cells (Fig. 1B and C). These results indicated that FoxM1 expression was effectively suppressed by the specific shRNA of FoxM1 in HeLa cells.

FoxM1 knockdown affects cell proliferation in vitro. To clarify whether or not FoxM1 downregulation can be a functional alteration, we examined cell viability by conducting MTT assay. We found that FoxM1 knockdown significantly inhibited cell growth from the second day (Fig. 1D).

FoxM1 downregulation promotes apoptosis. To evaluate the effect of FoxM1 knockdown on cell apoptosis, we investigated nuclear morphology by TUNEL and DAPI staining. In TUNEL-positive cells, nuclear condensation and fragmenta- tion representing apoptosis was observed. By contrast, the normal cells only showed blue DAPI-stained nucleus (Fig. 2A). The number of TUNEL-positive FoxM1 shRNA-transfected HeLa cells significantly increased compared with the control cells (Fig. 2B). Thus, FoxM1 knockdown markedly induced apoptosis of HeLa cells.

FoxM1 downregulation affects $u P A, M M P-2, M M P-9$ and $V E G F$ in vitro and in vivo. Several proteins that perform primary functions in the invasion, migration and metastasis of cervical cancer include uPA, MMP-2, MMP-9 and VEGF. Immunofluorescence analysis results demonstrated that FoxM1 downregulation decreased the expressions of uPA, MMP-2, MMP-9 and VEGF (Fig. 3A). This result is consistent with the signal differences in the fluorescence-labelled cells. We conducted qPCR and western blot analysis to determine whether or not the expression levels of uPA, MMP-2, MMP-9 

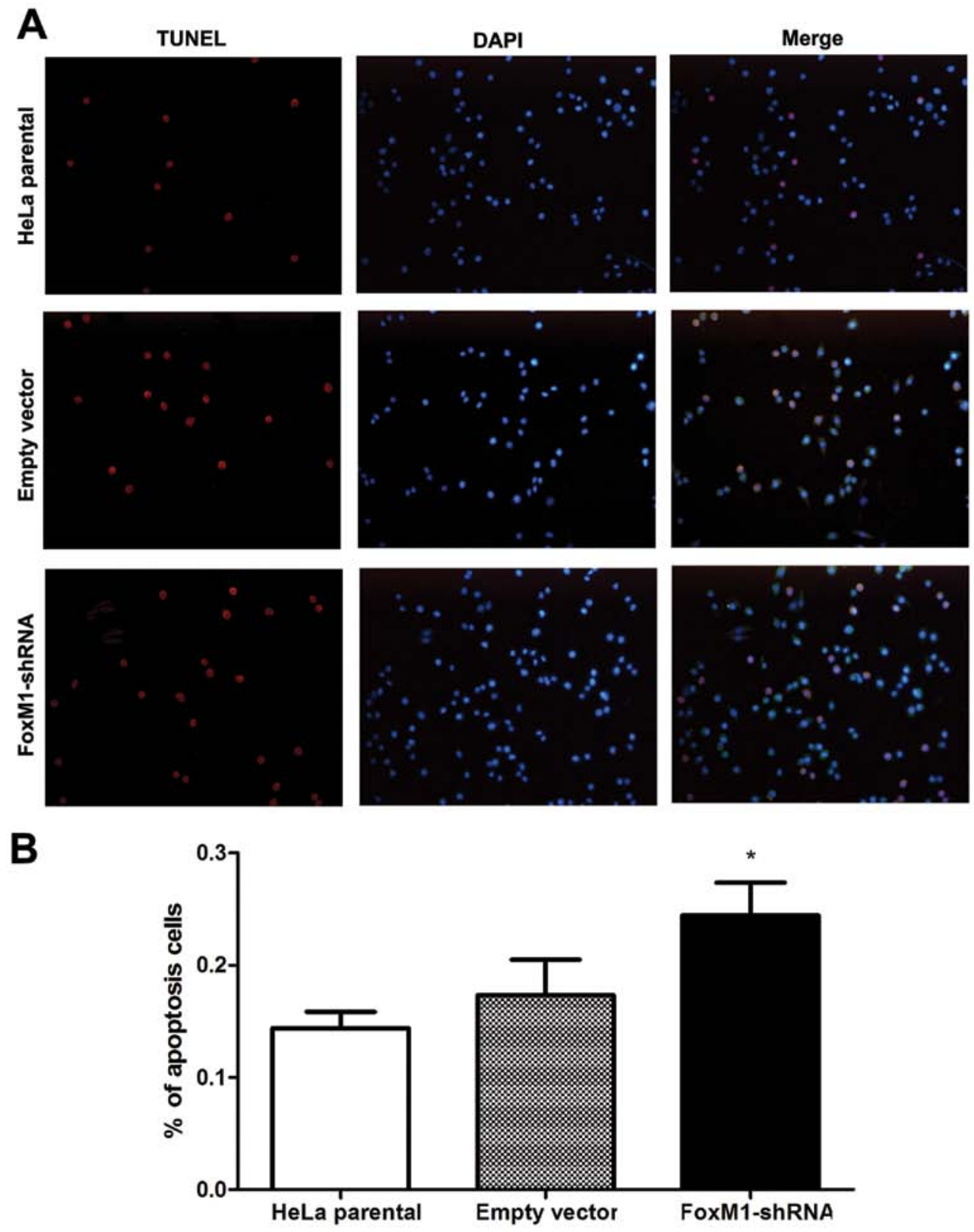

Figure 2. FoxM1-shRNA transfection inhibits apoptosis in HeLa cells. (A) FoxM1-shRNA stably transfected cells, empty vector-transfected cells and HeLa parental cells were stained with TUNEL-FITC. Nuclear morphology is indicated by DAPI and observed under a fluorescence microscope. For each cell type, TUNEL-positive cells were counted in five random fields at a magnification of x200. (B) A higher number of apoptotic FoxM1-shRNA transfected cells were detected than the two other cell types. TUNEL-positive cells were counted. Data are shown as mean \pm SEM from three independent experiments. " $\mathrm{P}<0.05$.

and VEGF are influenced by suppressed FoxM1 in HeLa cells. The mRNA and protein levels of uPA, MMP-2, MMP-9 and VEGF were markedly decreased in FoxM1 shRNA-transfected cells (Fig. 3B and C). We also conducted ELISA assays and observed similar patterns in the activities of uPA, MMP-2, MMP-9 and VEGF in stable-transfected cells (Fig. 3D-G). The primary FoxM1-shRNA xenografts were associated with the downregulation of these four factors at mRNA and protein levels (Fig. 4). These results suggested that the downregulation of FoxM1 inhibited uPA, MMP-2, MMP-9 and VEGF expressions in vivo and in vitro and prevented aggressive tumor invasion.

FoxM1 knockdown suppresses cell migration and invasion. FoxM1 downregulation inhibited the expression and impaired the activity of several important factors involved in tumor cell migration and invasion. We further examined whether or not FoxM1 downregulation affects cell invasion and migration ability by using a transwell system. The FoxM1 shRNAtransfected cells showed a low level of penetration into the membrane with (invasion) or without (migration) Matrigel compared with the control cells (Fig. 5). These results showed that Fox M1 downregulation notably suppressed cell migration and invasion.

FoxM1-shRNA group affects tumor growth in vivo. As expected, all of the three groups of cells developed tumors (Fig. 6A). The tumor growth curves showed that the growth pattern in the shRNA-FoxM1 group was significantly slower than that in the two control groups (Fig. 6B). The mice were 
A HeLa parental Empty vector FoxM1-shRNA
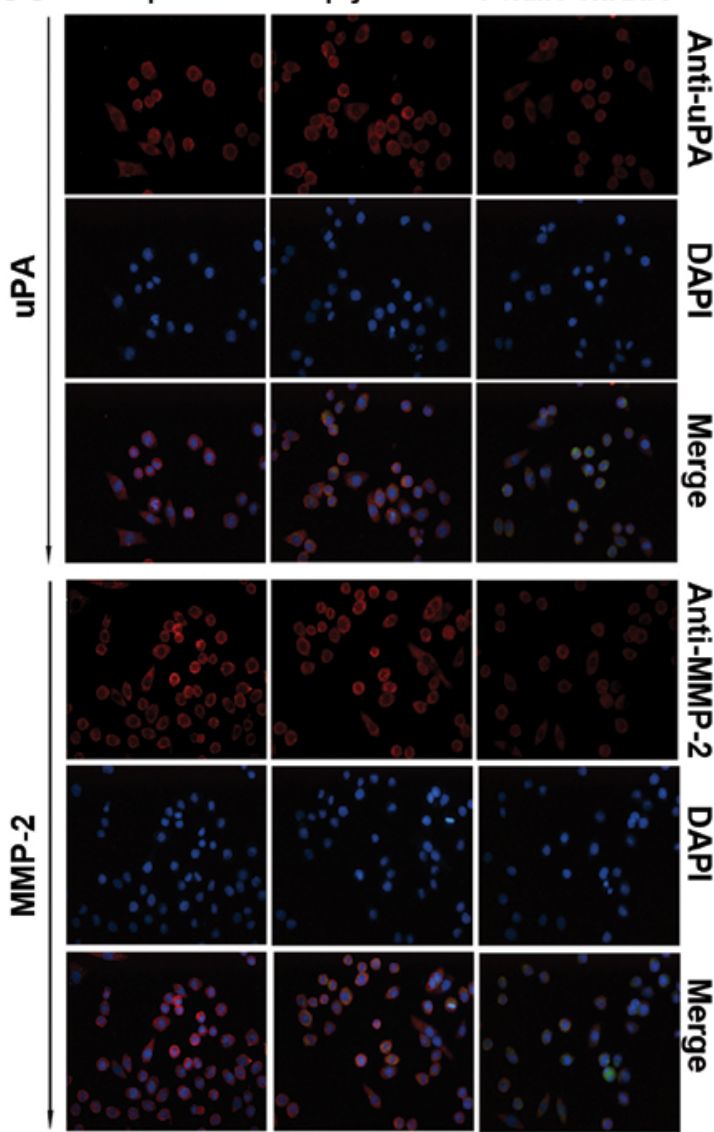

B

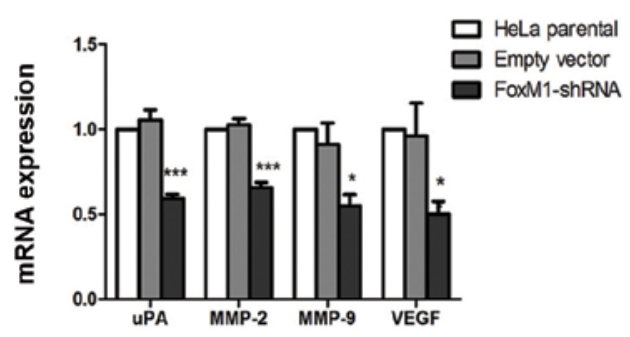

D

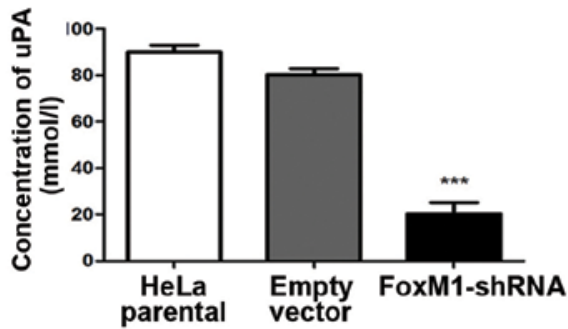

$\mathbf{F}$

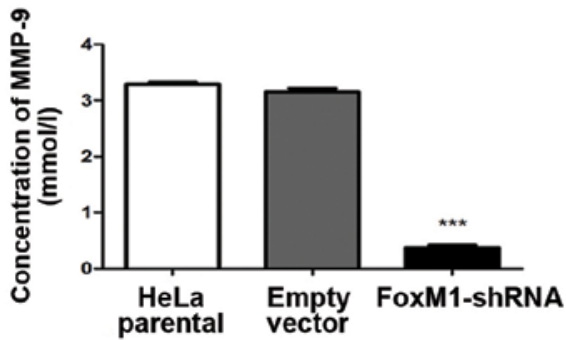

HeLa parental Empty vector FoxM1-shRNA
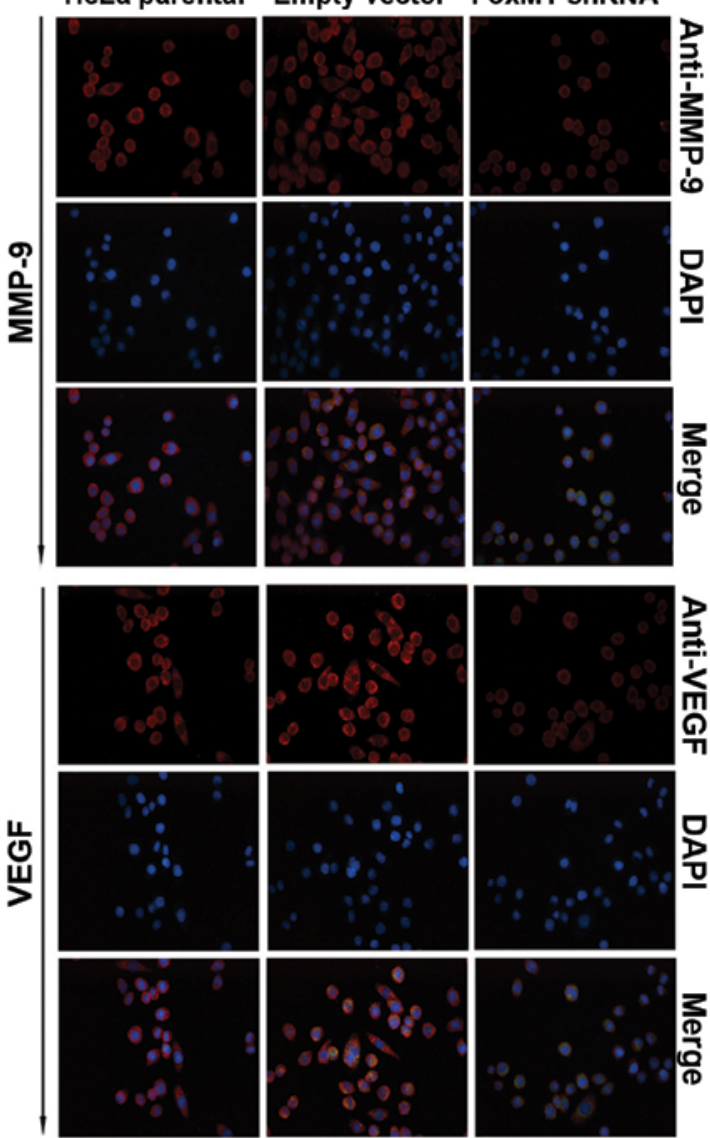

C

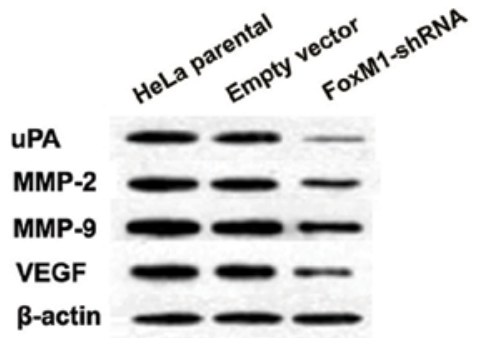

E

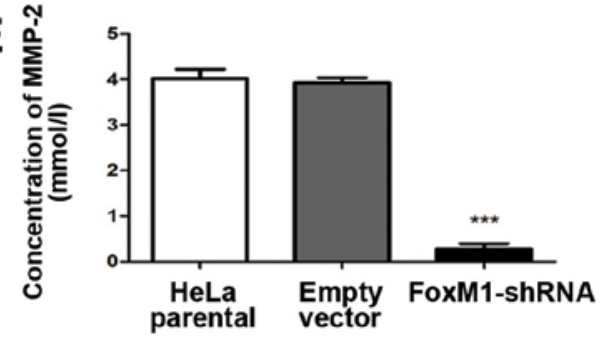

G

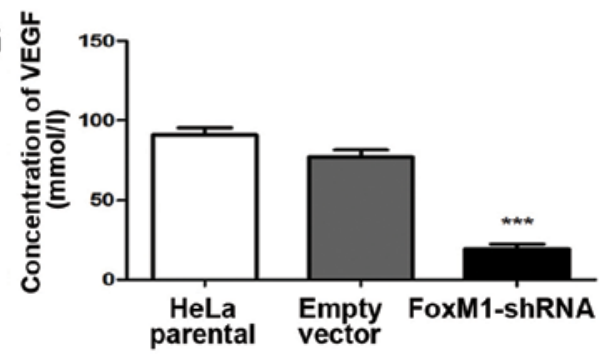

Figure 3. FoxM1 knockdown reduced the expression levels and activities of uPA, MMP-2, MMP-9 and VEGF in vitro. (A) The fluorescence signals of uPA, MMP-2, MMP-9 and VEGF were determined in cells after FoxM1 was downregulated. Merged images are the overlays of uPA, MMP-2, MMP-9 and VEGF red signals and nuclear staining by DAPI (blue). (B) Statistical diagrams showing the mRNA levels of uPA, MMP-2, MMP-9 and VEGF. (C) Protein levels of uPA, MMP-2, MMP-9 and VEGF in FoxM1-shRNA stably transfected cells, empty vector-transfected cells and HeLa parental cells were inspected by western blot analysis. (D-G) ELISA assay results showing that FoxM1 knockdown in HeLa cells inhibits the activities of uPA, MMP-2, MMP-9 and VEGF. Data are shown as mean \pm SEM from three independent experiments. ${ }^{* * *} \mathrm{P}<0.001$ compared with HeLa parental and/or empty vector-transfected cells. 

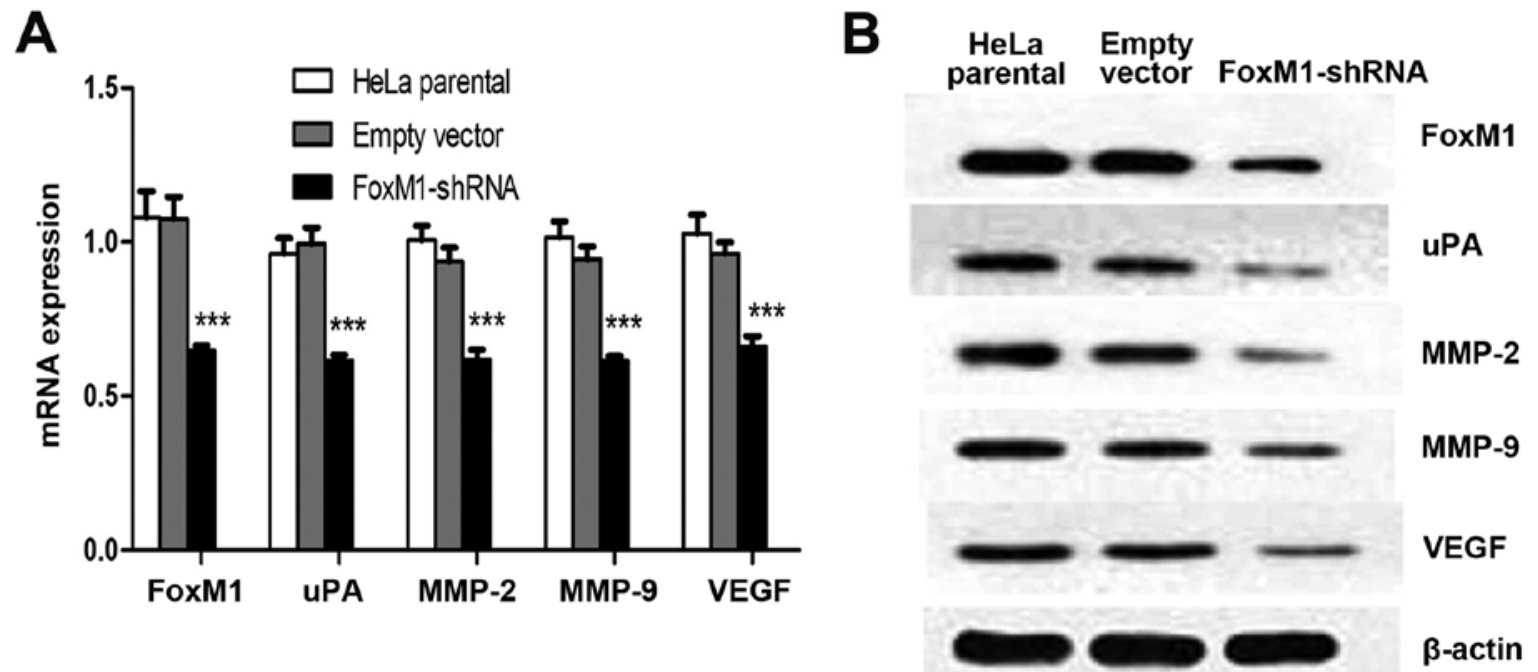

Figure 4. The mRNA and protein levels of uPA, MMP-2, MMP-9 and VEGF in FoxM1-shRNA stably transfected cells, empty vector-transfected cells and HeLa parental cells were inspected by qPCR and western blot analysis in vivo. ${ }^{* * *} \mathrm{P}<0.001$.

A
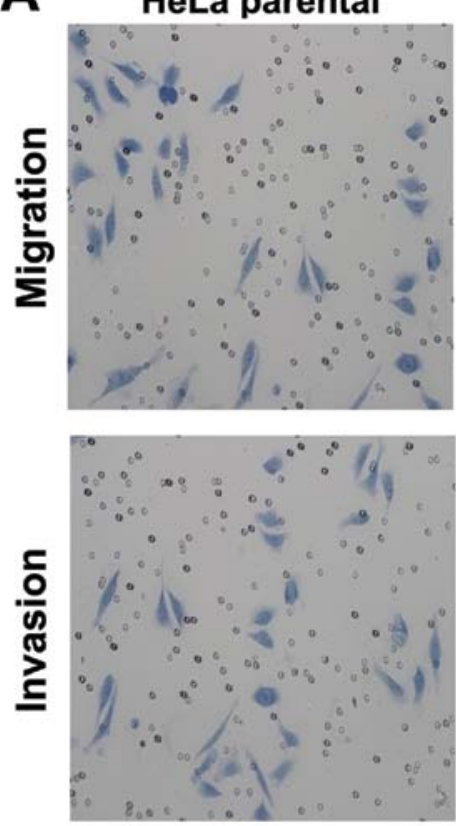

B

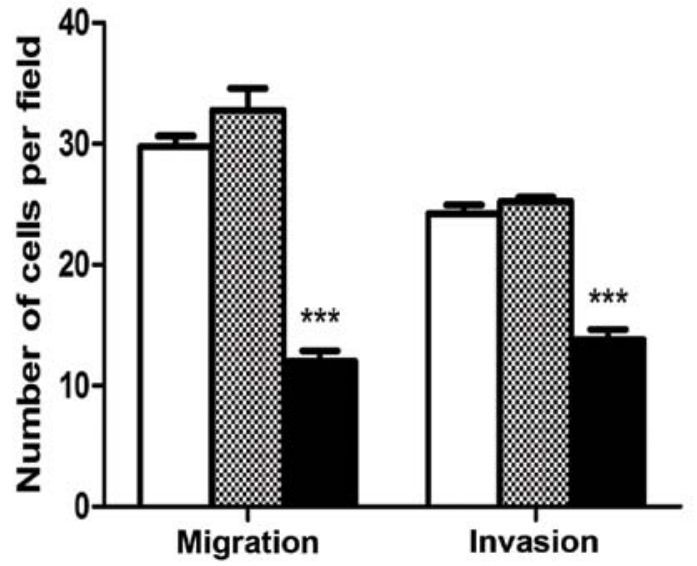

\section{FoxM1-shRNA}
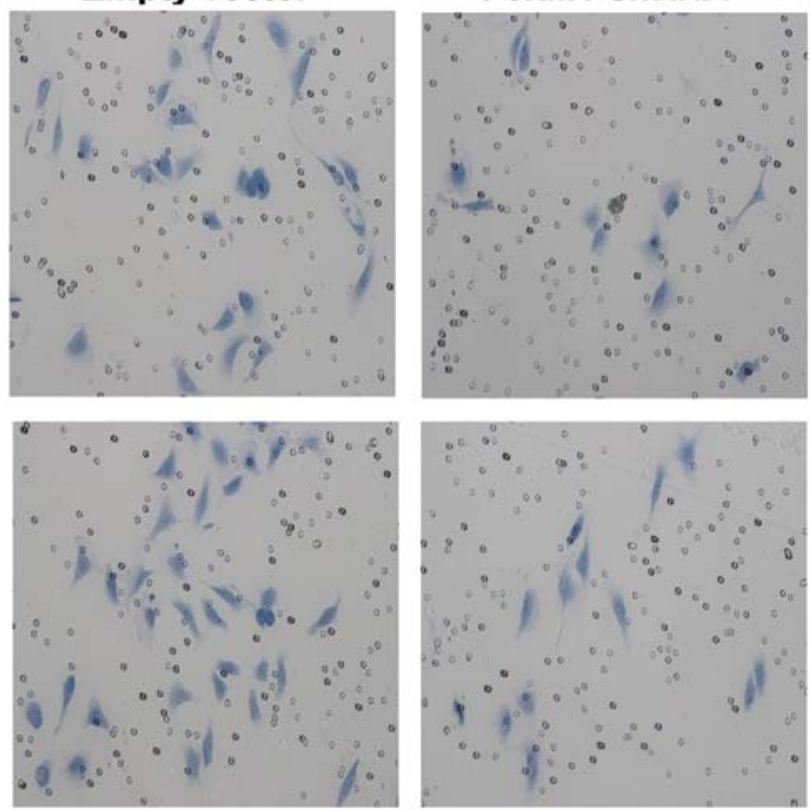

HeLa parental

Empty vector

FoxM1-shRNA

Figure 5. FoxM1 downregulation suppressed the migration and invasion ability of HeLa cells. (A) Membranes were stained with crystal violet and observed under a light microscope. (B) Statistical plots of the number of HeLa cells that penetrated the membranes as observed in migration assay and Matrigel invasion

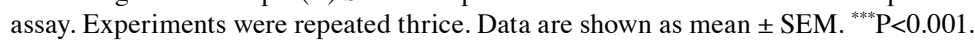


A

HeLa parental

FoxM1-shRNA

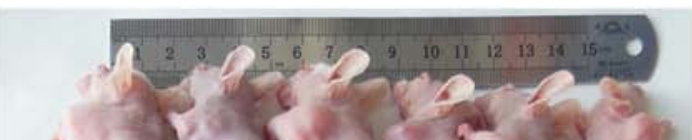

Empty vector

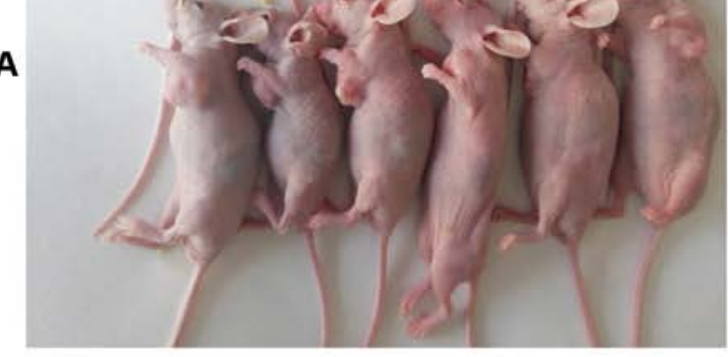

B

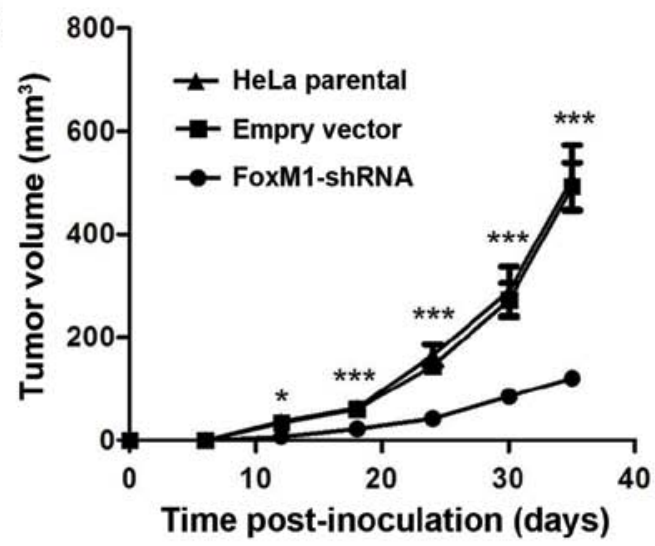

C

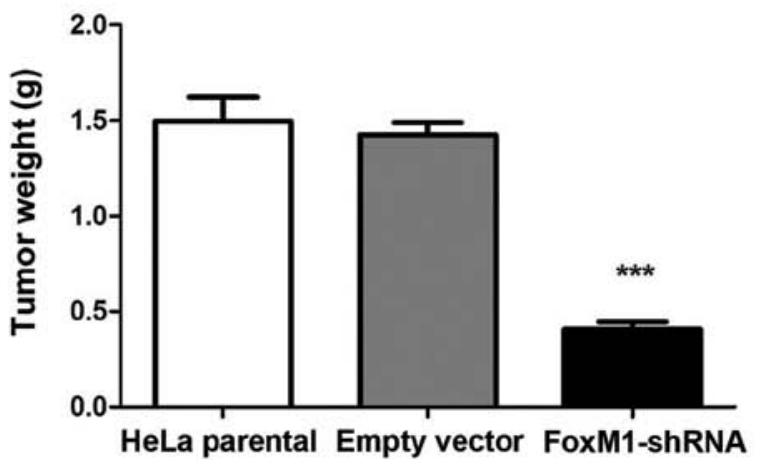

Figure 6. FoxM1 downregulation inhibited the growth of the transplanted tumor in nude mice. (A) After subcutaneous injection, tumors always formed, as shown in this image taken on day 35. (B) Tumor growth curves after injection in nude mice. Tumor volumes $\left(\mathrm{mm}^{3}\right)$ were measured at an interval of 6 days $(\mathrm{n}=6)$. (C) Weights of subcutaneous tumors in nude mice. The average tumor weight of parental HeLa, empty vector-transfected and FoxM1-shRNA cell groups were $1.497 \pm 0.309,1.425 \pm 0.157$ and $0.404 \pm 0.095 \mathrm{~g}$, respectively $(\mathrm{n}=6) .{ }^{* * * *} \mathrm{P}<0.001$ compared with parental HeLa and/or empty vector-transfected cells.

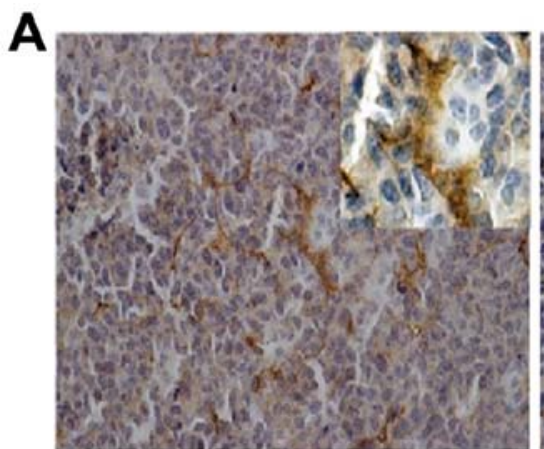

HeLa parental

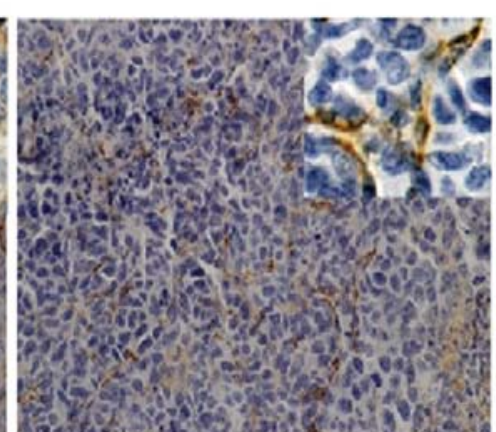

Empty vector

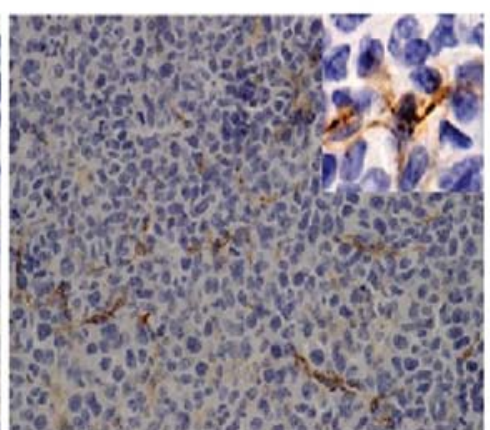

FoxM1-shRNA

B

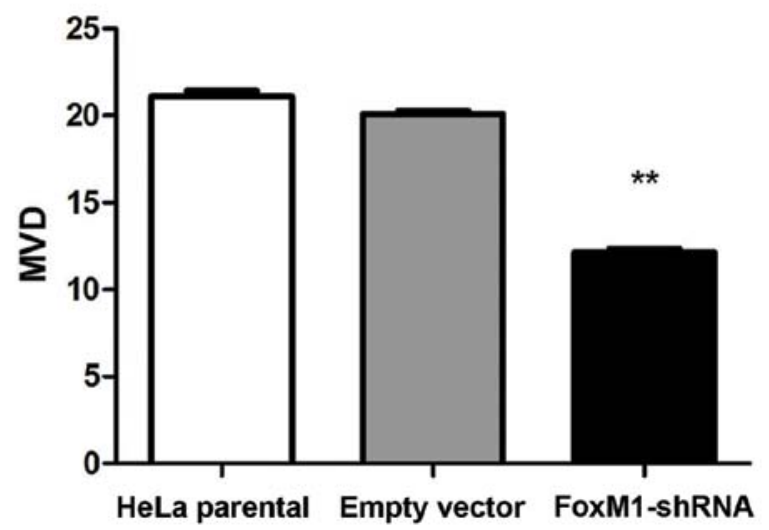

Figure 7. FoxM1 downregulation reduced angiogenesis in xenograft tumors. (A) Representative immunohistological staining of the xenografted tumor tissues generated by parental, empty vector and FoxM1-shRNA transfected HeLa cells (anti-CD31, original magnification, x400). (B) The number of capillaries was significantly lower in FoxM1-shRNA group compared with control. ${ }^{* * *} \mathrm{P}<0.01$. 
sacrificed at day 35 after injection. Tumor weight in the shRNA-FoxM1 group were significantly smaller than those in the parental HeLa group and the empty vector group (Fig. 6C). Tumor inhibitory rate in shRNA-FoxM1 group reached $72.76 \%$ compared with the empty vector group. No differences were observed between the two control groups in terms of growth pattern, tumor size and weight. These data indicated that the suppressed FoxM1 expression of HeLa cells could inhibit tumorigenicity in nude mice.

FoxM1 downregulation affects angiogenesis in xenograft tumors. Tumor induces angiogenesis to maintain the flow of nutrients for the increasing number of cells. To gain insights into tumor angiogenesis, we performed the MVD test. These results indicated that the number of capillary was significantly lower in the FoxM1-shRNA group compared with control group (Fig. 7). This result indicated a decrease in the angiogenic potential compared with the control groups.

\section{Discussion}

Tumorigenesis occurs as a result of excessive proliferation combined with reduced apoptosis (24). This factor is the particular target of FoxM1, a specific transcription factor. FoxM1 has been considered not only as a major regulatory factor in cell proliferation (25) but also as a potential inhibitor of cell apoptosis in malignancies $(26,27)$. In a previous study, FoxM1 was shown to bind and regulate a group of genes, which mainly participate in the control of late cell cycle events in G2 and M phases; binding is manipulated by the components of the homologous region of the gene involved in the cell cycle (28). In another study, DNA damage-induced apoptosis was induced by FoxM1 knockdown with RNAi or specific proteasome inhibitors (29). To elucidate the function of FoxM1 in the tumorigenesis of cervical cancers, we constructed specific shRNA and downregulated the expression of FoxM1 in HeLa cells with a relatively high endogenous expression of FoxM1. MTT assay and TUNEL assay results showed HeLa cells with attenuated proliferation and induced apoptosis. Considering the biological nature of these cells, we also found a similar pattern in our constructed nude mouse model. This result indicated that the volume and weight of the tumors were significantly decreased.

Angiogenesis is known as a pre-requisite process in tumor growth. Nevertheless, angiogenesis is based on the degradation of ECM components, including basement membrane collagen, and the release and/or activation of growth factors in MMPs, particularly MMP-2 and -9 (gelatinases A and B). In a multitude of malignancies, enhanced MMP-2 and -9 mRNA levels have been detected $(30,31)$. These factors directly participate in angiogenesis and metastasis as well as in clinical outcome and prognosis $(32,33)$. Other studies have revealed that a latent link is present between FoxM1 and MMPs $(6,8,26,34)$. For instance, Dai et al (35) found that FoxM1 promotes cellular invasiveness of glioma cells by upregulating MMP-2 and the relevant molecular mechanism involves the binding of FoxM1 and activating the promoter of the MMP-2 gene. The inhibition of MMP-2 and -9 expressions are attributed to the downregulation of FoxM1 in pancreatic cancer cells and vice versa (8). In our study, FoxM1 downregu- lation inhibited expression of MMP-2 and -9 at mRNA and protein levels in vitro and in vivo. This process also inhibited the activity of MMP-2 and -9 in the culture medium of HeLa cells. This result suggested a positive relationship between FoxM1 and MMPs (MMP-2 and -9).

The invasive ability of tumor cells consists of various aspects such as the uPA system, which consists of uPA and its specific cell surface receptor uPAR. The uPA level was upregulated in pancreatic cancer cells. In addition, the suppression of uPA-uPAR system results in the downregulation of angiogenin and decrease in angiogenic potential in vitro and in vivo (36). In glioblastoma, a similar pattern can be observed in angiogenesis after uPA and UPAR are inhibited (37). In other studies, cell viability and cell invasion ability are significantly reduced after instantaneous uPA silencing is conducted. Furthermore, decreased tumor growth and survival rates are observed in an orthotopic mouse prostate cancer model (38). However, the information describing the mechanism by which FoxM1 interacts with uPA in cervical cancer cells remains incomplete. In the present study, immunofluorescence analysis, real-time PCR, western blot analysis and ELISA assay results showed that the levels and activities of uPA decreased after FoxM1 was downregulated compared with the control cells.

Various growth factors, which have been identified as critical regulators of angiogenesis and tumor invasion, are observed in the degradation of ECM. VEGF is considered as the main angiogenic activator produced and secreted by cancer cells (39). The autocrine/paracrine action of VEGF can also promote tumor growth independent of angiogenesis (40). Angiogenesis is a process by which new blood vessels grow and can be observed in physiological and pathological events (41). Angiogenesis occurs when tumor reaches a diameter ranging from 1 to $2 \mathrm{~mm}$, thereby leading to tumor growth and metastasis (42). Zhang et al (43) demonstrated that FoxM1 functions as an angiogenic switch in tumors by transcriptionally activating VEGF expression and directly binding to the Forkhead binding elements (FHRE) of VEGF promoter in glioma cells. A PB-Cre/Foxm1 fl/fl /TRAMP transgenic mouse model in which FoxM1 is efficiently deleted has been established, indicating a marked decrease in the mRNA of VEGF-A as shown by real-time PCR. The siRNA-mediated depletion of FoxM1 is also observed in TRAMP C2 mouse prostate adenocarcinoma cells. However, aberrant angiogenesis does not occur (44). In clear cell renal cell carcinoma, the overexpression of FoxM1 was determined at mRNA and protein levels. The aberrant expression and activity of VEGF and angiogenesis are detected after FoxM1 is downregulated (34). Similar outcomes can be observed in gastric cancer cells (17). This result is consistent with our study, in which the reduced levels and activities of VEGF as well as the number of microvessels in the xenograft tumors induced FoxM1 expression.

We observed that FoxM1 downregulation resulted in reduced expressions and activities of uPA, MMP-2, MMP-9 and VEGF. Considering this result, we evaluated the effects of FoxM1 downregulation on the migration and invasion of HeLa cells. We found that the ability of FoxM1-shRNA transfected cells to migrate and invade the Matrigel was remarkably weakened compared with that of the control cells. Therefore, these results indicated that the knockdown of FoxM1 inhibits 
the aggressiveness of cervical cancer possibly by regulating uPA, MMP-2, MMP-9 and VEGF.

Previous studies demonstrated the associations among uPA, MMP-2, MMP-9 and VEGF. For example, a significant association between uPA and MMP-2 expression $(\mathrm{P}=0.028)$ is found in cervical intraepithelial neoplasia, which is regarded as pre-cancerous lesions of cervical cancers (45). Fang et al (46) verified that the inhibition of specific MMP-2 results in a decrease in angiogenic and proteolytic activities of tumor nodules and restricts tumor growth by $70 \%$ in vivo. Fang et al (46) also demonstrated that the activated MMP-2 on the cell surface of endothelial cells can interact with integrin $\alpha v \beta 3$, which is highly expressed in melanoma metastases, to promote angiogenesis and vice versa (47). Tumor cell-induced angiogenesis is also observed in Ad-MMP-2-infected lung cancer cells, and this abrogation of MMP-2 resulted in the reduced tumor growth and formation of lung nodules in mice (48). In another study, the inactivation of MMP-2 transcriptional level reduces integrin $\alpha v \beta 3$, PI3K/AKT-induced VEGF expression, thereby decreasing tumor cell-induced angiogenesis (49). Raghu et al (37) observed the UPA and UPAR shRNA-mediated inhibition of angiogenesis; this process can be attributed to the enhanced SVEGFR1 secretion independent of GM-CSF but dependent on TIMP-1 in endothelial and glioblastoma cells. Although, He et al (50) has reported the aggressive function of FOXM1 in cervical cancer via MMP-2/9 and relative signal pathways, we have demonstrated the function of FoxM1 in proliferation, apoptosis, migration, invasion and angiogenesis more systematically. While the detailed mechanisms by which FoxM1 acts on these cells and the reciprocity among these four factors in cervical cancers should be further investigated.

\section{References}

1. Siegel R, Ma J, Zou Z and Jemal A: Cancer Statistics, 2014. CA Cancer J Clin 64: 9-29, 2014.

2. Pectasides D, Kamposioras K, Papaxoinis G and Pectasides E: Chemotherapy for recurrent cervical cancer. Cancer Treat Rev 34: 603-613, 2008.

3. Li S, Hu T, Lv W, et al: Changes in prevalence and clinical characteristics of cervical cancer in the People's Republic of China: a study of 10,012 cases from a nationwide working group. Oncologist 18: 1101-1107, 2013.

4. Yee GP, de Souza P and Khachigian LM: Current and potential treatments for cervical cancer. Curr Cancer Drug Targets 13 205-220, 2013

5. Ahmad A, Wang Z, Kong D, et al: FoxM1 down-regulation leads to inhibition of proliferation, migration and invasion of breast cancer cells through the modulation of extra-cellular matrix degrading factors. Breast Cancer Res Treat 122: 337-346, 2010.

6. Kim IM, Ackerson T, Ramakrishna S, et al: The Forkhead Box $\mathrm{m} 1$ transcription factor stimulates the proliferation of tumor cells during development of lung cancer. Cancer Res 66: 2153-2161, 2006.

7. Liu M, Dai B, Kang SH, et al: FoxM1B is overexpressed in human glioblastomas and critically regulates the tumorigenicity of glioma cells. Cancer Res 66: 3593-3602, 2006.

8. Wang Z, Banerjee S, Kong D, Li Y and Sarkar FH: Downregulation of Forkhead Box M1 transcription factor leads to the inhibition of invasion and angiogenesis of pancreatic cancer cells. Cancer Res 67: 8293-8300, 2007.

9. Feng Y, Wang L, Zeng J, et al: FoxM1 is overexpressed in Helicobacter pylori-induced gastric carcinogenesis and is negatively regulated by miR-370. Mol Cancer Res 11: 834-844, 2013.

10. Kalinichenko VV, Major ML, Wang X, et al: Foxm1b transcription factor is essential for development of hepatocellular carcinomas and is negatively regulated by the p19 $9^{\mathrm{ARF}}$ tumor suppressor. Genes Dev 18: 830-850, 2004.
11. Chan DW, Yu SY, Chiu PM, et al: Over-expression of FOXM1 transcription factor is associated with cervical cancer progression and pathogenesis. J Pathol 215: 245-252, 2008.

12. Guan P, Chen H, Li HJ, Duan J and Chen JY: Expression and significance of FOXM1 in human cervical cancer: a tissue micro-array study. Clin Invest Med 34: E1-E7, 2011.

13. Khongkow P, Karunarathna U, Khongkow M, et al: FOXM1 targets NBS1 to regulate DNA damage-induced senescence and epirubicin resistance. Oncogene: Oct 21, 2013 (Epub ahead of print). doi: 10.1038/onc.2013.457.

14. Raychaudhuri P and Park HJ: FoxM1: a master regulator of tumor metastasis. Cancer Res 71: 4329-4333, 2011.

15. Li Y, Ligr M, McCarron JP, et al: Natura-alpha targets forkhead box $\mathrm{m} 1$ and inhibits androgen-dependent and -independent prostate cancer growth and invasion. Clin Cancer Res 17: 4414-4424, 2011.

16. Sun H, Teng M, Liu J, et al: FOXM1 expression predicts the prognosis in hepatocellular carcinoma patients after orthotopic liver transplantation combined with the Milan criteria. Cancer Lett 306: 214-222, 2011.

17. Li Q, Zhang $\mathrm{N}$, Jia Z, et al: Critical role and regulation of transcription factor FoxM1 in human gastric cancer angiogenesis and progression. Cancer Res 69: 3501-3509, 2009.

18. Wierstra I and Alves J: FOXM1, a typical proliferation-associated transcription factor. Biol Chem 388: 1257-1274, 2007.

19. Wang IC, Chen YJ, Hughes D, et al: Forkhead box M1 regulates the transcriptional network of genes essential for mitotic progression and genes encoding the SCF (Skp2-Cks1) ubiquitin ligase. Mol Cell Biol 25: 10875-10894, 2005.

20. Laoukili J, Kooistra MR, Bras A, et al: FoxM1 is required for execution of the mitotic programme and chromosome stability. Nat Cell Biol 7: 126-136, 2005.

21. Cao Y, E G, Wang E, et al: VEGF exerts an angiogenesisindependent function in cancer cells to promote their malignant progression. Cancer Res 72: 3912-3918, 2012.

22. Naito S, von Eschenbach AC, Giavazzi R and Fidler IJ: Growth and metastasis of tumor cells isolated from a human renal cell carcinoma implanted into different organs of nude mice. Cancer Res 46: 4109-4115, 1986.

23. Kim YH, Kim MA, Park IA, et al: VEGF polymorphisms in early cervical cancer susceptibility, angiogenesis, and survival. Gynecol Oncol 119: 232-236, 2010.

24. Green DR and Evan GI: A matter of life and death. Cancer Cell 1: 19-30, 2002.

25. Katoh M and Katoh M: Human FOX gene family (Review). Int J Oncol 25: 1495-1500, 2004

26. Ahmed M, Uddin S, Hussain AR, et al: FoxM1 and its association with matrix metalloproteinases (MMP) signaling pathway in papillary thyroid carcinoma. J Clin Endocrinol Metab 97: E1-E13, 2012.

27. Uddin S, Ahmed M, Hussain A, et al: Genome-wide expression analysis of Middle Eastern colorectal cancer reveals FOXM1 as a novel target for cancer therapy. Am J Pathol 178: 537-547, 2011.

28. Chen X, Muller GA, Quaas M, et al: The forkhead transcription factor FOXM1 controls cell cycle-dependent gene expression through an atypical chromatin binding mechanism. Mol Cell Biol 33: 227-236, 2013.

29. Halasi M and Gartel AL: Suppression of FOXM1 sensitizes human cancer cells to cell death induced by DNA-damage. PLoS One 7: e31761, 2012.

30. Kallakury BV, Karikehalli S, Haholu A, Sheehan CE, Azumi N and Ross JS: Increased expression of matrix metalloproteinases 2 and 9 and tissue inhibitors of metalloproteinases 1 and 2 correlate with poor prognostic variables in renal cell carcinoma. Clin Cancer Res 7: 3113-3119, 2001.

31. Maatta M, Soini Y, Liakka A and Autio-Harmainen H: Differential expression of matrix metalloproteinase (MMP)-2, MMP-9, and membrane type 1-MMP in hepatocellular and pancreatic adenocarcinoma: implications for tumor progression and clinical prognosis. Clin Cancer Res 6: 2726-2734, 2000.

32. Klein G, Vellenga E, Fraaije MW, Kamps WA and de Bont ES: The possible role of matrix metalloproteinase (MMP)-2 and MMP-9 in cancer, e.g. acute leukemia. Crit Rev Oncol Hematol 50: 87-100, 2004.

33. Passlick B, Sienel W, Seen-Hibler R, et al: Overexpression of matrix metalloproteinase 2 predicts unfavorable outcome in early-stage non-small cell lung cancer. Clin Cancer Res 6: 3944-3948, 2000. 
34. Xue YJ, Xiao RH, Long DZ, et al: Overexpression of FoxM1 is associated with tumor progression in patients with clear cell renal cell carcinoma. J Transl Med 10: 200, 2012.

35. Dai B, Kang SH, Gong W, et al: Aberrant FoxM1B expression increases matrix metalloproteinase-2 transcription and enhances the invasion of glioma cells. Oncogene 26: 6212-6219, 2007.

36. Gorantla B, Asuthkar S, Rao JS, Patel J and Gondi CS: Suppression of the uPAR-uPA system retards angiogenesis, invasion, and in vivo tumor development in pancreatic cancer cells. Mol Cancer Res 9: 377-389, 2011.

37. Raghu H, Nalla AK, Gondi CS, Gujrati M, Dinh DH and Rao JS UPA and UPAR shRNA inhibit angiogenesis via enhanced secretion of SVEGFR1 independent of GM-CSF but dependent on TIMP-1 in endothelial and glioblastoma cells. Mol Oncol 6: 33-47, 2012.

38. Pulukuri SM, Gondi CS, Lakka SS, et al: RNA interferencedirected knockdown of urokinase plasminogen activator and urokinase plasminogen activator receptor inhibits prostate cancer cell invasion, survival, and tumorigenicity in vivo. J Biol Chem 280: 36529-36540, 2005.

39. Zhao R, Liu XQ, Wu XP, et al: Vascular endothelial growth factor (VEGF) enhances gastric carcinoma invasiveness via integrin alpha(v)beta6. Cancer Lett 287: 150-156, 2010.

40. Lee J, Lee J, Yu H, Choi K and Choi C: Differential dependency of human cancer cells on vascular endothelial growth factormediated autocrine growth and survival. Cancer Lett 309: 145-150, 2011.

41. Risau W: Mechanisms of angiogenesis. Nature 386: 671-674, 1997.

42. Folkman J: Angiogenesis. Annu Rev Med 57: 1-18, 2006.
43. Zhang Y, Zhang N, Dai B, et al: FoxM1B transcriptionally regulates vascular endothelial growth factor expression and promotes the angiogenesis and growth of glioma cells. Cancer Res 68: 8733-8742, 2008.

44. Cai Y, Balli D, Ustiyan V, et al: Foxm1 expression in prostate epithelial cells is essential for prostate carcinogenesis. J Biol Chem 288: 22527-22541, 2013.

45. No JH, Jo H, Kim SH, et al: Expression of MMP-2, MMP-9, and urokinase-type plasminogen activator in cervical intraepithelial neoplasia. Ann NY Acad Sci 1171: 100-104, 2009.

46. Fang J, Shing Y, Wiederschain D, et al: Matrix metalloproteinase-2 is required for the switch to the angiogenic phenotype in a tumor model. Proc Natl Acad Sci USA 97: 3884-3889, 2000.

47. Silletti S, Kessler T, Goldberg J, Boger DL and Cheresh DA: Disruption of matrix metalloproteinase 2 binding to integrin alpha vbeta 3 by an organic molecule inhibits angiogenesis and tumor growth in vivo. Proc Natl Acad Sci USA 98: 119-124, 2001.

48. Chetty C, Bhoopathi P, Joseph P, Chittivelu S, Rao JS and Lakka S: Adenovirus-mediated small interfering RNA against matrix metalloproteinase-2 suppresses tumor growth and lung metastasis in mice. Mol Cancer Ther 5: 2289-2299, 2006.

49. Chetty C, Lakka SS, Bhoopathi P and Rao JS: MMP-2 alters VEGF expression via alphaVbeta3 integrin-mediated PI3K/AKT signaling in A549 lung cancer cells. Int J Cancer 127: 1081-1095, 2010.

50. He SY, Shen HW, Xu L, et al: FOXM1 promotes tumor cell invasion and correlates with poor prognosis in early-stage cervical cancer. Gynecol Oncol 127: 601-610, 2012. 